ÉGYPTE monde arabe

\section{Égypte/Monde arabe}

$24 \mid 1995$

Anthropologies de l'Égypte 1

\title{
Pour une approche anthropologique de la réislamisation
}

Saadia Radi

\section{OpenEdition}

1 Journals

Édition électronique

URL : https://journals.openedition.org/ema/622

DOI : $10.4000 /$ ema.622

ISSN : 2090-7273

Éditeur

CEDEJ - Centre d'études et de documentation économiques juridiques et sociales

Édition imprimée

Date de publication : 31 décembre 1995

Pagination : 13-28

ISSN : 1110-5097

\section{Référence électronique}

Saadia Radi, « Pour une approche anthropologique de la réislamisation », Égypte/Monde arabe [En ligne], 24 | 1995, mis en ligne le 08 juillet 2008, consulté le 07 juillet 2022. URL : http:// journals.openedition.org/ema/622 ; DOI : https://doi.org/10.4000/ema.622

Ce document a été généré automatiquement le 7 juillet 2022.

Tous droits réservés 


\title{
Pour une approche anthropologique de la réislamisation
}

\author{
Saadia Radi
}

1 L'« islamisme » - la volonté affichée de réislamiser la société - est sans doute l'aspect le plus marquant de l'évolution actuelle des sociétés musulmanes et des débats qui y ont cours ${ }^{1}$. Certes, leur dynamique économique et leur insertion dans la société mondiale sont des aspects non moins importants de cette évolution, mais il est clair que ces aspects contribuent à la tonalité générale des débats en question ; sans doute même en sont-ils l'origine. Nombre d'ouvrages sont consacrés/depuis environ deux décennies, à la question de l'islamisme, les auteurs insistant sur les rapports du politique, et du religieux, sur les acteurs engagés dans le processus et sur certains groupes dont on considère qu'ils occupent une place particulière dans les projets de réislamisation : les femmes ou les minorités non musulmanes.

2 Cette littérature riche et prolifique a toutefois tendance à négliger ce que Mauss nommait «l'expérience des catégories »: elle ne s'intéresse guère à la façon dont les acteurs - engagés ou non - considèrent, à partir de leur vie quotidienne, le thème de la réislamisation. Or, une juste compréhension du contenu et de la portée de celle-ci ne peut faire l'impasse sur cette perception. La référence à l'islam, le succès d'une certaine forme d'austérité musulmane symbolisée par le port du voile, l'acquiescement à l'application de la loi islamique (sharî̀a), sont des attitudes difficiles à évaluer si l'on ne tient pas compte de l'expérience subjective des musulmans. Cette expérience est complexe, différente d'un groupe à l'autre comme d'une personne à l'autre, car le partage d'une référence n'implique pas, comme l'a bien montré Kertzer², le partage de son contenu. Trop d'études, en effet, sans doute influencées par la tradition orientaliste, tentent de construire un contenu commun aux multiples références à l'islam, alors qu'il vaudrait sans doute mieux comprendre la logique sociale de cette multiplicité.

3 Mais là aussi, l'absence d'“ expérience des catégories » rend difficile l'accès à la logique sociale et favorise le discours sur le discours, la tentation de raccorder les références actuelles de l'islam à l'histoire savante de la pensée musulmane comme le fait, par 
exemple, Olivier Carré quand il définit les islamistes comme des «néo-hanbalites déviants $»^{3}$. Il n'est pas aisé de dire en quoi cette définition - mélange entre les catégories orientalistes et les catégories des ulémas - éclaire le développement contemporain du thème de la réislamisation. Elle décrit l'objet de l'extérieur comme si les acteurs n'avaient rien à y faire.

4 M'écartant de cette façon de procéder, j'ai choisi d'adopter une approche anthropologique qui s'intéresse d'abord à la façon dont les musulmans construisent de l'intérieur - et dans la diversité - les références à l'islam, envisagent le thème de l'austérité et l'application de la sharîa. Plusieurs raisons ont déterminé mon choix : la volonté d'éviter une extériorité qui tend généralement à exagérer la singularité des sociétés musulmanes; le désir de rendre justice au point de vue des acteurs en restituant, autant que faire se peut, la complexité mais aussi l'indécision de leur univers; le souci de ne pas confondre discours politique, sentiment religieux, évaluation morale et respect des convenances suivant l'idée - bien trop répétée ça et là - selon laquelle, en islam, tout serait lié et, surtout, le politique et le religieux.

5 J'ai aussi choisi de m'intéresser aux acteurs non engagés, considérant qu'il n'était pas possible de décrire la dynamique sociale et la réception du thème de la réislamisation en basant mon travail sur l'attitude et les conduites de ses partisans les plus actifs. Plutôt que sur l'émission, j'ai donc travaillé sur la réception, sur les raisons pour lesquelles des thèmes comme le port du voile ou l'application de la sharî'a pouvaient être envisagés sous un jour favorable sans impliquer un engagement individuel fort ou un positionnement politique marqué. Là aussi, la notion de solidarité sans consensus, de partage de la référence sans partage du contenu, m'a été particulièrement utile. Sans doute cette solidarité doit-elle beaucoup à l'existence d'un habitus, au sens de Bourdieu (1980); elle n'est pas pour autant l'expression du maintien d'une culture religieuse traditionnelle.

6 Le détour par «l'expérience des catégories " s'avère très utile à ce propos puisqu'il permet de rendre compte des ruptures dans l'imagination religieuse: la façon de s'affirmer musulman aujourd'hui traduit, en effet, bien plus l'aspect religieux d'une identité contemporaine largement définie hors de la religion, que la conséquence d'une identité religieuse qui se transmettrait à travers le temps. "Être musulman » est une chose qui varie, comme varie ce que l'on entend par être un homme ou par avoir une vie heureuse. Certes, des rites et des symboles se transmettent, mais ils sont largement réinterprétés, quand bien même ils continuent à imposer un jeu de distinctions. Il est, par exemple, impossible de décrire le port du niqâb (voile dissimulant entièrement le visage. N.D.L.R.) comme la simple continuation du port du voile au début du siècle ou dans les années soixante, même si l'un et l'autre s'autorisent d'une lecture interprétative de la même sourate.

7 La démarche anthropologique possède des avantages mais également certaines contraintes, la principale étant qu'elle implique de travailler sur des groupes réduits. C'est certainement là contrepartie de "l'expérience des catégories»: celle-ci est difficile à envisager sans une véritable "compréhension de l'intérieur» - toujours suivant Mauss -, c'est-à-dire sans une fréquentation assidue du groupe sur lequel on travaille. Ceci en limite évidemment la taille. Mais on ne le reprocherait sans doute pas à la discipline si la généralisation des enquêtes statistiques et de leur version popularisée - les sondages d'opinion - n'avait établi l'idée de « représentativité de l'échantillon ». Paradoxalement, l'historien est rarement pris à partie sur ce fait alors 
qu'il travaille sur des témoignages bien plus limités et incomplets que n'importe quel anthropologue ne le fera jamais. Il y a dans cette question de la représentativité, quand elle est soulevée, une évidente volonté d'invalider non pas la démarche mais ses résultats, c'est-à-dire les conclusions que l'anthropologue en tire. Généralement, plus la question est polémique, plus le problème de la représentativité apparaît. En fait, la validité de la démarche anthropologique tient à ce qu'elle décrit, certes, des individualités (des individus aussi bien que des groupes), mais des individualités socialement situées, c'est-à-dire intégrées à un système social et qui n'est lui-même qu'un agrégat d'individualités semblables. De ce principe d'interdépendance, il résulte que la discipline ne se préoccupe pas de monades, d'êtres incommensurables, mais d'acteurs sociaux partageant au minimum les critères qu'ils utilisent pour se différencier les uns des autres.

8 J'ai mené mon travail de terrain à l'université du Caire auprès d'étudiants et d'étudiantes de troisième et quatrième année du département de sciences politiques et de la section «études islamiques » de la faculté des lettres (35 entretiens, dont la plupart enregistrés). J'ai ainsi bénéficié de l'avantage de travailler au sein d'un milieu - l'université - jusqu'à un certain point socialement hétérogène, ce qui me renseignait au moins partiellement sur le caractère partagé d'un certain type de rapport à la thématique de la réislamisation. Certes, je ne prétends pas - et le paragraphe qui précède en témoigne - avoir eu affaire à un milieu représentatif de l'ensemble des tendances observables dans la société égyptienne ; en revanche, je crois que ce terrain, singulier comme tous les terrains anthropologiques, permet de comprendre, sur le rapport au réfèrent religieux, un certain nombre d'éléments qui ne sont pas réductibles à ses spécificités. Travailler avec des étudiants présente des avantages dont le moindre n'est certainement pas celui d'assurer l'observateur de la contemporanéité du système de références des acteurs auxquels il s'intéresse. L'enquête à proprement parler a duré plus de neuf mois et a commencé avec difficulté.

Quand est-on islamiste?

9 L'un des premiers entretiens recueillis a bouleversé d'emblée un certain nombre des catégories que j'avais à l'esprit et a rendu définitivement délicate l'utilisation du terme « islamiste » :

«Le but de tout musulman est l'application de la sharîa, la réforme de la société, de l'économie et de la politique. Pour cela, il faut appliquer la sharî'a d'une manière juste et non fallacieuse. Il n'y a pas, dans le monde musulman, un seul pays qui l'applique vraiment; il n'existe que des applications formelles, comme en Arabie Saoudite. Appliquer la sharî'a de manière juste, c'est se conformer aux prescriptions du Coran, de la Sunna... Dans la sharî'a, il y a les hudûd (châtiments corporels. N.D.L.R.). Si on les applique, la société devient pieuse. Les hudûd empêchent de nombreux crimes. Si les gens savaient que s'ils tuent, ils vont être tués, ils ne commettraient pas de crimes. Face aux différences entre les classes, l'islam a introduit la zakât (aumône légale. N.D.L.R.). On prend au riche pour donner au pauvre, cela assure l'équité. Il n'y a pas de différence entre le religieux et le politique. Aucun système n'a jamais réalisé l'équité, à part l'islam quand il était appliqué, du Prophète à Atatürk. Si l'on appliquait la sharî'a, les crimes se feraient rares, on n'aurait presque plus besoin d'utiliser les hudûd. On n'a qu'à prendre en exemple la période du Prophète. Dans les autres systèmes politiques, de nombreux crimes sont commis. Il existe dans le monde divers systèmes, mais pas de stabilité. 
11 «La religion musulmane est une, bien qu'il y ait plusieurs écoles; les désaccords ne portent pas sur le fond mais sur les détails. À partir du moment où l'on est d'accord sur le fond, la sharî'a est claire. L'Arabie Saoudite et l'Iran n'appliquent pas la sharî'a. Les gamâ'ât (groupes islamistes. N.D.L.R.) sont le résultat des conditions imposées par la société. Quand ils [les membres des gamâ'ât] constatent que la sharî'a n'est pas appliquée et qu'on applique des lois qui viennent de l'étranger, quand ils constatent qu'il n'y a ni justice ni stabilité, ils demandent qu'on applique la sharî́a. Nous vivons dans la corruption, les crimes, l'adultère.

«Par ailleurs, on essaie de donner une mauvaise image de l'islam et des musulmans. Face à cela, il y a trois attitudes. Certains jeunes acceptent la situation et ne s'intéressent pas à l'islam. D'autres veulent le changement et ont recours à la prédication $\left(d a^{\prime} w a\right)$ écrite ou orale, pour dire aux gens ce qui est licite et illicite. D'autres encore affrontent l'État et utilisent là violence. Ils ne font que se venger : si le pouvoir tue l'un des leurs, ils tuent un homme du pouvoir. Je pense qu'ils ont tort quand ils tuent et posent des bombes : ils peuvent toucher des personnes innocentes. Dernièrement, les attentats à la bombe ont augmenté ; je crôis que leurs auteurs sont étrangers. Ils font ça pour qu'on accuse les groupes islamistes. Ces derniers commettent aussi des actes terroristes, mais c'est seulement par vengeance. Je ne leur donne pas raison.

13 «Les gamâ'ât veulent appliquer la sharî'a. Ils ne veulent pas de lois étrangères à l'islam. Les Frères musulmans utilisent la parole et l'écrit. Je suis proche d'eux. Je suis proche de toute gamâ'a qui n'utilise pas la violence et qui ne traite pas la société d'impie (kâfira), comme les gens du groupe al-Takfir wa-l-hijra qui te traitent d'impie si tu ne pries pas à l'heure exacte. La seule chose qui soit intolérable est le polythéisme. L'islam est une religion de tolérance, alors que le groupe al-Takfir wa-l-hijra considère comme impies tous les gens qui ne lui sont pas rattachés.

14 «À l'université, j'ai rencontré des étudiants appartenant aux gamâ'ât. Certains habitent à côté de moi à la cité universitaire, ou étudient avec moi. Nous avons d'abord eu des relations banales, puis nous avons commencé à discuter de religion. Ils ne demandent pas aux gens de faire partie de leurs groupes. Ils prêchent seulement : s'ils voient que quelqu'un ne prie pas, ils lui expliquent pourquoi il faut prier ; s'ils voient un étudiant passer beaucoup de temps avec une étudiante, ils lui expliquent qu'il ne faut plus le faire. Ils ne m'ont jamais demandé d'être des leurs, mais ils essayent, indirectement, de me faire apprécier leurs idées. Ils prononcent aussi des prêches (khutba) à la mosquée de l'université. Le plus important pour eux, c'est le commandement du bien et le pourchas du mal. Ils demandent aux étudiants de prier, de jeûner, de s'éloigner de tout ce qui peut déplaire à Dieu. En même temps, s'ils voient quelqu'un consommer de l'alcool, ils l'en empêchent en jetant l'alcool; ils peuvent même le frapper. Or je pense qu'un individu n'a pas le droit de punir.» (Muhammad, 22 ans, section «études islamiques ", $3^{\mathrm{e}}$ année)

Comment situer l'auteur de ce discours? Il n'est pas politiquement engagé en ce sens qu'il n'appartient pas à un groupe d'acteurs engagés. Il n'est pas membre d'une gamâ'a et désapprouve l'usage de la violence. Mais son discours n'est pas, pour autant, un discours quiétiste ; c'est au contraire un discours axé sur la réforme de la société. On peut ainsi être favorable à l'application de la sharî'a, à la réforme de la société par l'islam et, en même temps, non engagé politiquement, c'est-à-dire n'œuvrant pas à l'intérieur d'un groupe militant pour la réislamisation. Peut-on alors considérer cette 
posture comme relevant de l'«islamisme »? François Burgat avait noté la différence entre fondamentalisme et islamisme, arguant $d u$ fait que le fondamentalisme n'établissait pas d'emblée un rapport entre l'immoralité et le système économique et politique ${ }^{4}$. Or, il est clair que l'étudiant interviewé plus haut établit un rapport entre islam et justice économique, islam et régimes politiques et que la réforme morale - le commandement du bien et le pourchas du mal - est envisagée comme devant déboucher sur la réforme sociale. En même temps, la relative distance qu'il marque visà-vis des gamâ'ât, sa condamnation de la violence (envers l'État comme envers les individus) et son insistance à considérer l'unité des musulmans, l'éloignent de l'engagement politique. Penser l'islam comme réforme de la société (c'est-à-dire politiquement, au sens large du terme) n'implique pas le passage à l'engagement politique.

Cette position met en porte-à-faux d'autres conceptions comme celles défendues, en Égypte, par Muhammad Saïd al-'Ashmawi ${ }^{5}$. Celui-ci, on le sait, considère que la revendication d'application de la sharî'a est une demande politique dans la mesure où la législation égyptienne lui est conforme, à l'exception des hûdud qui sont inapplicables (et, dans la conception d'al-'Ashmawi, n'ont pas été édictés pour être appliqués). Dans une perspective historique, ce point de vue peut être défendu avec une certaine vraisemblance, puisque c'est un qâdi, membre important des Frères musulmans, 'Abd al-Qâdir 'Uda qui, le premier, dans l'Égypte contemporaine, formula comme revendication politique l'application de la sharî'a. Mais l'étudiant que je viens de citer n'est pas engagé dans un processus de contestation du régime ni même affilié à des groupes dont on pourrait penser qu'ils œuvrent en ce sens. Le passage à l'action politique est autre chose que le désir de voir appliquer la sharî'a et d'y voir une solution crédible aux problèmes présents de la société. Il semble au contraire que cette conception de la réislamisation soit, en partie, dépolitisée. Arrêtons-nous un instant sur cette question.

Réforme et dépolitisation

17 J'entends par « dépolitisation » le refus de considérer que la réforme de la société par la réislamisation passe par une forme d'activisme politique, voire de confrontation avec le pouvoir :

Je connais les groupes islamistes depuis que j'ai seize ans, c'est-à-dire depuis que je sais ce qu'est la réalité. Ce qui me plaît chez eux, c'est qu'ils veulent le changement (...) Pourquoi l'Amérique intervient-elle dans ce que font les pays musulmans ? Les gamâ'ât savent ces choses-là. (...) Ce qui me déplaît chez eux, c'est quand ils disent que le pouvoir est impie. (Mahmûd, 23 ans, section « études islamiques », $3^{\mathrm{e}}$ année). Selon moi, les gamâ'ât sont bien. Il faut qu'ils éveillent la conscience des gens mais sans utiliser la violence. S'ils veulent que l'Égypte devienne du jour au lendemain comme l'Arabie Saoudite, ce n'est pas possible. Leur premier rôle est de montrer aux gens ce qu'est la vraie religion et de les guider. La politique, c'est autre chose. On doit s'en préoccuper quand tout le monde devient conscient. Dans ce pays, il y a beaucoup de corruption, chacun cherche son propre intérêt. (...) Si on applique l'islam maintenant, il y a des gens qui ne seront pas d'accord et on aura la même situation qu'au Liban. (Dinâ, 22 ans, $3^{\mathrm{e}}$ année de sciences politiques).

La plupart des étudiants interrogés et qui évoquent la nécessité de réislamiser la société distinguent la situation intérieure de l'Égypte et la situation de l'islam dans le monde de l'activisme politique, considéré comme un ferment de désunion entre musulmans. Il peut, certes, s'agir d'une forme de prudence - la crainte de parier " politique» - mais bien souvent cette prudence est argumentée, nourrie d'exemples 
sur les dangers de la division ${ }^{6}$, et ressemble davantage à une position personnelle qu'à un discours de façade. Il semble évident que ce qui est nommé "politique " n'a rien à voir avec des préoccupations de type social ni avec une volonté d'appliquer la sharî'a. Ces dernières ne paraissent pas relever de la « politique » dans la mesure où elles sont perçues comme non conflictuelles : conflit et politique, chez les étudiants interrogés, sont liés. Ce qui n'empêche pas certains d'entre eux de souhaiter que le religieux gouverne le politique, sans pour autant mettre en avant l'application de la sharî'a.

L'islam est religion et comportement. Si l'on introduisait la religion dans la politique, ce serait mieux. Aujourd'hui, nous vivons dans la corruption, le piston (wasta). N'arrivent aux fonctions politiques que les personnes qui en bénéficient. Si l'on applique l'islam, les comportements vont changer et la société sera équitable. (...) Actuellement, je ne veux pas appliquer la sharî́a mais si tout le monde l'applique, je l'appliquerai. (Iman, 23 ans, $3^{\mathrm{e}}$ année de sciences politiques).

D'autres étudiants considèrent que les groupes islamistes tiennent des discours éloignés de la vie quotidienne, à connotation politique ou religieuse trop marquée, et qu'ils perdent beaucoup de temps et d'énergie à se disputer le "leadership » :

Ce que je n'aime pas non plus chez certains groupes, c'est l'agression et le meurtre. L'islam n'a jamais ordonné de tuer. On doit pratiquer l'islam individuellement, mais eux procèdent autrement : ils obligent les gens à faire telle ou telle chose et traitent de problèmes sans intérêt - par exemple, si tu es en prison, est-ce que tu peux faire les prières aux toilettes? Tout ça, c'est loin des problèmes quotidiens. Est-ce qu'ils ont résolu ces problèmes pour se poser de telles questions? Ils devraient expliquer aux gens comment se comporter au travail, à la maison, expliquer la parole de Dieu. Même dans les mosquées, les thèmes qu'ils abordent sont loin des préoccupations des gens, ce sont des questions de pouvoir, de leadership, ou des explications du Coran. Leurs désaccords font d'eux des partis politiques. ('Ali, 24 ans, $3^{\mathrm{e}}$ année de sciences politiques)

La religion sans quiétisme ni fondamentalisme, les affaires de la société sans politique, l'application de la sharî'a sans contestation du pouvoir, la volonté de réislamisation sans engagement actif dans les gamâ'ât: la thématique de la réislamisation n'est pas une affaire d'opposants ni de fondamentalistes. Ce que cette forme de " dépolitisation » permet de mettre en évidence, c'est la possibilité, pour les acteurs en présence, de situer hors d'une perspective conflictuelle des discours comme ceux portant sur la nécessaire application de la sharî'a, quand bien même ces discours caractérisent-ils également des groupes engagés dans une démarche effectivement politique. Bien souvent, les étudiants se distinguent de ces derniers en les accusant de diviser les musulmans, de recourir à la violence ou au terrorisme.

Ce discours ne doit pas nous induire en erreur en suggérant une disjonction entre l'action des groupes islamistes et l'attitude des étudiants qui n'en font pas partie; d'abord parce qu'il se présente, en général, comme la critique de certaines gamâ'ât et non pas de toutes, ni même de la majorité d'entre elles; ensuite, parce qu'il ne met nullement en cause l'accord sur la thématique de la réislamisation elle-même et, bien au contraire, montre que celle-ci est commune à des acteurs engagés et à des acteurs qui ne le sont pas, voire répugnent à l'être, commune également à des acteurs qui tentent de vivre de façon austère - les membres des gamâ'ât - et à d'autres qui envisagent seulement de le faire. L'insistance sur le caractère «non politique » de la réislamisation illustre ainsi la diffusion d'une thématique - et, d'une certaine manière, sa banalisation - puisqu'elle cesse d'être liée à des groupes précis pour devenir, exprimée dans des termes semblables, l'affaire de tous, une façon « normale » d'évaluer 
ce qui se passe dans la société égyptienne. En ce sens, le terme de "dépolitisation " n'est pas un euphémisme: il nous indique seulement que la critique des gouvernants impies et illégitimes - du point de vue démocratique ${ }^{7}$ ou du point de vue islamique ne constitue pas le fond du problème. Celui-ci serait plutôt la réforme de la société par la réforme de soi.

Dans la perspective que je viens rapidement d'indiquer, le terme «islamiste » n'est d'aucune utilité descriptive : ou il ne désigne que les acteurs engagés et occulte le fait que les acteurs non engagés partagent les mêmes conceptions quant à la réforme de la société, ce qui n'est pas satisfaisant du point de vue de l'analyse politique; ou il désigne à la fois les uns et les autres, mais alors sa capacité descriptive est des plus réduites. Cela nous suggère aussi que les jeux d'oppositions ne sont pas simplement externes entre partisans et adversaires de la réislamisation, par exemple - mais aussi et surtout internes. La perception des gamâ'ât qui ressort de ces entretiens est très contrastée et se rapporte principalement à un univers fragmenté. Quand 'Ali affirme que les gamâ'ât se disputent le « leadership », il témoigne simplement du fait que l'unicité du thème (la réislamisation) ne permet pas de supposer l'unité des acteurs.

Le pieux et le puritain

Les étudiants interrogés opèrent une distinction très nette, parmi les acteurs de la réislamisation, entre le pieux (multazim) qui respecte scrupuleusement les préceptes de la religion, et le puritain (mutazammit) :

Certains garçons et filles des gamâ'ât sont religieux et pieux (multazimîn). On se comporte avec eux normalement. Du côté des garçons, il y a un vrai respect des prescriptions. Ils nous saluent (al-salâmu 'alaykum) sans nous serrer la main, je préfère ça. Il y a aussi des puritains (mutazammitîn) qui n'ont même pas de relations avec les pratiquants (mutadayyîn) comme eux. Ils restent seuls pour ne pas commettre de péché. Certaines filles pratiquantes (mutadayyinât) donnent des conseils. Quand je suis mal habillée - que je porte un décolleté -, elles me montrent ce que je dois faire. Elles prient pour que Dieu m'indique le bon chemin. Elles me disent des choses que je ressens profondément. (...)

Une personne qui n'est pas religieuse ne peut jamais être bien car elle est possédée par Satan, qui fait tout ce qu'il faut pour la garder. Mais celui qui fait au moins la prière, c'est-à-dire celui qui n'est pas très pratiquant, est aussi quelqu'un de bien. Évidemment, il y a une différence entre celui qui pratique vraiment, celui qui pratique un peu et celui qui ne pratique pas du tout. Moi, je prie, je fais le Ramadan, ça ne veut pas dire que je suis religieuse. Je commets des fautes, mais pas des fautes graves. Celui qui ne prie pas et consomme de l'alcool peut commettre n'importe quelle faute. Je souhaite l'application de la sharî'a mais sans fanatisme (ta'assub), sans en rajouter dans la religion. Il y a des gens [les mutazammitîn] qui comprennent mal la religion. Ils croient que la tolérance est une erreur (khalat); quand ils sourient, ils croient qu'ils commettent un péché; pour eux, la religion est fanatisme, la musique est illicite. Elle l'est seulement si elle empêche de prier. Lorsqu'on prie et qu'on jeûne, la musique demeure une nourriture pour l'âme. Aller à la mer en maillot est illicite, mais pas la musique. » (Sawsan, 24 ans, $3^{\mathrm{e}}$ année de sciences politiques).

Pour 'Ali, la distinction est encore plus radicale :

Ce qui est important, ce n'est pas le port du voile (higâb) ou de la gallabeya, c'est le fait d'orienter les gens selon les règles de l'islam. Il faut qu'ils deviennent conscients et réalisent la richesse et l'importance de l'islam. Il faut appliquer l'islam d'une façon progressive. Quand la femme et l'homme sont conscients, ils se conduisent comme il convient. La femme met le voile d'elle-même et respecte tout ce qui va avec. Il faut des étapes pour appliquer la sharî́a. il y a des musulmans 
pieux (multazimîn) qui respectent les préceptes de l'islam et se comportent correctement avec les gens. Il y a aussi des puritains (multazammitîm) qui n'évoluent pas avec leur époque, qui vivent un islam rigide. Mais dans l'islam, il y a de la souplesse. ('Ali, 24 ans, $3^{\mathrm{e}}$ année de sciences politiques)

Nous avons ici affaire à un type de critique lié à la dépolitisation. Ce qui est en cause, une fois de plus, n'est pas la conception de la réislamisation mais la façon d'y parvenir et le type d'hommes et de femmes dont l'engagement peut servir d'exemple. Si l'austérité semble parfaitement acceptée comme idéal de comportement - Sawsan n'est pas choquée qu'une étudiante pieuse la conseille sur sa manière de s'habiller -, la façon de s'y prendre pour l'instaurer n'est pas exempte de polémiques. Le style sévère des membres de certaines gamâ'ât est pris à partie, comme leur isolement vis-à-vis de la société. Le conseil est valorisé par rapport à l'usage de la violence, le changement dans la durée par rapport au changement immédiat. L'austérité mesurée du croyant l'emporte sur la sévérité excessive du puritain. Au demeurant, en insistant sur l'aspect rebutant de certaines attitudes - dont celle qui consiste à considérer tous ceux qui ne sont pas puritains comme des impies (kufâr) -, les étudiants ne mettent pas en cause l'activité des gamâ'ât en général mais seulement l'activité de certaines d'entre elles, et encore reconnaissent-ils parfois que leurs critiques contre celles-ci reposaient sur un préjugé :

Mon premier contact avec les gamâ'ât a eu lieu pendant les élections à l'université. J'étais avec des gens qui sont contre les groupes islamistes. Au cours des élections, nous étions en train de discuter avec eux quand ils ont commencé à réciter des sourates du Coran. L'un d'eux est venu me demander de lire le Coran. Je me suis mise en colère, je lui ai répondu que personne n'avait le droit de m'imposer de réciter le Coran. Je le récite quand je veux. Je lui ai dit: «Tu n'es pas musulman mais extrémiste.» II est resté calme et il a commencé à discuter avec moi. Franchement, je n'ai pas su quoi répondre. J'avais honte car je comprenais qu'il avait raison. Il m'a expliqué que les mauvais musulmans et les extrémistes n'étaient pas dans leur groupe. (Chérifa, 21 ans, $3^{\mathrm{e}}$ année de sciences politiques)

D'un entretien à l'autre, la conception du multazim se précise. Elle n'est pas politique mais éthique ; elle est liée à sa conduite, à sa capacité à comprendre autrui et à l'aider plutôt qu'à le juger. L'austérité ne parait donc pas antithétique avec la tolérance. Elle n'implique pas un certain modèle d'autorité - une relation de commandement, en quelque sorte - mais une relation de conseil et d'éducation. Que le crédit moral qui accompagne la pratique personnelle de l'austérité et la maîtrise du conseil crée une relation inégalitaire entre un "aîné » (tout au moins par la maturité) et un "cadet » (par l'imperfection de son comportement) ne peut être interprété comme l'acceptation d'un principe d'autorité dans la conduite de sa vie personnelle. Bien au contraire, les étudiants insistent sur l'importance de l'absence de contrainte dans le choix de se conformer fidèlement à l'islam. Cette attitude peut sembler assez opportuniste et renvoyer à une stratégie de visibilité. Mais la critique soutenue des puritains et de ceux qui veulent « appliquer l'islam tout de suite » indique à l'évidence que les qualités des multazimîn ne sont pas seulement destinées à faire valoir l'islam. Elle révèle dans quelles limites le processus de réislamisation est acceptable et le type d'acteurs qui ne peuvent espérer y participer. Elle souligne que l'islam a d'abord une portée éthique dont est garante l'attitude individuelle des croyants. D'une certaine manière - quoi qu'il en soit des liens évidents que l'on peut établir entre politique et religion -, cette présence de l'éthique et du comportement exemplaire au sein de la vie sociale rappelle que la référence à la religion n'est pas réductible au politique. Ainsi les catégories 
servant à évaluer la qualité de l'engagement dans le processus de réislamisation sontelles des catégories éthiques. De ceci non plus la notion d'«islamisme» ne rend pas compte, en se focalisant sur la compétition pour le pouvoir.

Les « terroristes » et la dépolitisation

Les "terroristes" (irhâbiyyîn) ne sont pas des types moraux bien dessinés et sont d'ailleurs difficiles à situer. Peu d'étudiants les situent dans les gamâ'ât et certains établissent même une distinction marquée entre les deux: "Les terroristes n'appliquent pas l'islam, car l'islam ne dit pas que le pouvoir est impie. (...) Je parle des terroristes, pas des gamâ'ât » ('Azîz, 23 ans, section " études islamiques », $3^{\mathrm{e}}$ année). On peut, certes, considérer qu'entre la vigueur des propos sur la réislamisation et la violence des «terroristes", il existe une relation qui ne peut être ignorée (Roussillon, 1994) mais la critique de la violence - à l'instar de la critique du puritanisme témoigne aussi du refus de la contrainte et de la compétition pour le pouvoir :

Il y a aussi les terroristes (irhâbiyyîn), mais je préfère le terme d'«extrémiste" (mutatarrif), qui désigne précisément l'homme qui voit un chemin mais s'éloigne de ce chemin. C'est celui qui a la violence pour méthode, qui oblige autrui à être comme lui. Le puritain (mutazammit), lui, veut appliquer l'islam sans aucune souplesse ni adaptation à l'époque actuelle. Quant au terroriste (irhabî), c'est celui qui fait des fatâwî (plur. de fatwa) et qui applique l'islam en ayant recours à la violence. (Gamâl, 24 ans, $3^{e}$ année de sciences politiques)

Nous avons affaire ici à un dispositif homologue - mais inverse - à celui utilisé par les adversaires de la réislamisation, qui la lient strictement au terrorisme. De manière polémique, l'évocation du "terrorisme " sert à qualifier une pratique austère de l'islam, à la définir comme dangereuse en l'assimilant au terrorisme ou à exclure le terrorisme du champ - légitime, lui - de la réislamisation. Dans les deux cas, les attaches sociales du mouvement doivent être gommées pour ne pas contredire ou atténuer la portée de la démonstration. Si l'on suit le débat public égyptien sur cette question, il est clair que la tendance générale consiste à séparer "terrorisme " et "réislamisation", cette dernière étant un objectif trop partagé pour donner lieu à polémique. De plus, cette distinction permet aisément de délégitimer l'action des "terroristes ", alors que lier les deux termes délégitimerait la réislamisation. Affirmer que le « terrorisme » - c'est-à-dire l'action violente - est la seule chose illégitime dans le programme des gamâ'ât, faire porter la condamnation sur la violence et, particulièrement, sur la violence envers les innocents, revient du même coup à valider tout le reste (y compris le puritanisme). C'est d'ailleurs la position qui fut prise, en 1993, par le "Comité des religieux pour la réforme » - lagna al-'ulamâ' li-l-islâh ${ }^{8}$ - qui se proposait de mettre fin à la violence mais ne la considérait pas comme liée à la réislamisation, bien au contraire :

Nous vivons à l'heure de tous les désordres. (...) Un croyant qui s'avise de se laisser pousser la barbe conformément aux ordres du Prophète se voit accuser d'être un voyou, un malade ou un terroriste... Toute croyante qui décide de porter le voile de tête (...) ou le voile intégral devient le symbole de la réaction et de l'arriération. (...) Une campagne sans merci prend pour cible la sharî́a tandis qu'une guerre également sans merci est livrée contre l'islam... (cité et traduit par Roussillon, 1994, p. 317).

Pour autant, il ne faut pas négliger le fait que certains étudiants ne condamnent pas entièrement le passage à la violence et considèrent que les gamấât se livrent effectivement au terrorisme, même s'ils quittent alors le registre de la réislamisation : 
L'islam est là pour résoudre les problèmes, mais les responsables [des gamâ'ât] ne le comprennent pas ainsi. Au début, j'étais très proche des groupes islamistes, même quand ils agressaient des policiers ou s'attaquaient aux symboles du pouvoir. Quand ils s'en sont pris à des innocents, je me suis éloigné d'eux. C'est la police qui gouverne; elle se comporte très mal. Quand un groupe l'attaque, elle se venge. Je me suis éloigné des groupes quand ils ont tué la petite Shayma, et aussi quand j'ai su qu'ils volaient des bijoutiers. (Farîd, 22 ans, section "études islamiques ", $3^{\mathrm{e}}$ année)

La possibilité de distinguer terrorisme et réislamisation n'exclut donc pas de porter un jugement relativement favorable à certaines formes de violence, même si l'on admet qu'elles relèvent de l'excès. Si les étudiants interrogés se refusent à considérer que le pouvoir est kâfir, il ne faut pas en déduire qu'ils lui accordent une grande légitimité. Ce refus découle de celui de la violence, non de l'opinion flatteuse qu'ils auraient du régime. Sur ce point, ils peuvent d'ailleurs tenir des propos totalement dénués d'ambiguïté : «Le terrorisme et l'État sont deux maux entre lesquels on se refuse à choisir. » Les nombreuses évocations de la situation libanaise - «si l'on impose l'islam d'un coup, l'Égypte deviendra un second Liban » - donnent un sens particulièrement lourd à la dépolitisation que j'évoquais plus haut. C'est l'idée même de « changer par la politique » qui est, d'une certaine manière, en crise, d'où l'insistance sur le conseil et l'explication: "La politique c'est autre chose, on ne s'en occupera que lorsque tout le monde sera conscient. » ('Umar, 23 ans, $3^{\mathrm{e}}$ année de sciences politiques) Le retour à l'islam doit précéder le passage au politique :

Si les gens s'améliorent en appliquant les préceptes de l'islam, il y a des gens bien qui se présenteront aux élections législatives, et ceux qui se seront améliorés grâce à l'islam voteront pour eux. S'ils appliquent l'islam véritable, peut-être un jour l'un d'eux deviendra-t-il président de la République. (Sami, 21 ans, $2^{\mathrm{e}}$ année de sciences politiques)

Le «terrorisme » comme catégorie du discours sert ainsi peut-être davantage à penser la dangerosité du politique plutôt que sa primauté et permet de situer la réislamisation dans le domaine de la réforme sociale par la réforme de soi plutôt que par le passage au politique. Il faut, de ce point de vue, tenir compte de la simultanéité, dans les discours sur la réislamisation, de la critique des gouvernants et de la critique de la démocratie. La mise en cause de cette dernière ne doit pas être simplement interprétée comme l'un des éléments de la rhétorique " anti-occidentale ». Quand les étudiants affirment que la démocratie ne serait pas bonne pour l'Égypte car elle a rendu possible, en Occident, l'immoralité et l'impiété, ce discours renvoie aussi à l'impossibilité du politique à constituer des individus moraux.

C'est en cela que l'enracinement de la réforme dans la réislamisation, c'est-à-dire dans une tradition religieuse (certes réinventée), prend toute sa valeur, car la religion ne dispense pas seulement une explication du monde, elle propose aussi, comme l'a montré Geertz ${ }^{9}$, des modèles pour l'action dotés d'une forte puissance émotionnelle. Le multazim est très certainement l'un de ces modèles. L'idée qu'il importe avant tout de constituer des individus moraux pour que la société guérisse de ses maux introduit le parfum spécifique du religieux dans un espace normalement dévolu au politique. On ne gagne n'en à rationaliser ce caractère propre de la réislamisation en le réduisant à une instrumentalisation du langage religieux pour évoquer des revendications politiques, comme l'ont fait nombre d'auteurs. Si les étudiants éprouvent le besoin de parier de la société égyptienne en des termes empruntés à la religion, il faut donc admettre qu'ils ne le font pas sans de bonnes raisons et que le choix du vocabulaire est aussi une 
évaluation de la nature du problème. Ce n'est pas leur prêter une piété étonnante que de considérer que la réislamisation est pour eux une solution parce qu'il s'agit précisément d'un programme religieux et d'une attitude morale. Religion ne veut pas dire, ici, soumission à la transcendance mais possibilité de devenir un certain type d'homme et un certain type de femme, par rapport à un certain état de choses. Sans doute est-il aisé de passer de ce souci éthique à une forme d'action politique. Pourtant, le passage n'implique pas que l'action politique soit la continuation normale du souci éthique. Il est nécessaire d'insister sur cela si l'on veut éviter de se méprendre sur le sens exact de l'attitude des étudiants: surévaluer la probabilité de leur engagement comme sous-estimer leur intérêt pour le débat politique. En même temps, il paraît évident que le souci éthique est le terreau du passage au politique, c'est-à-dire le lieu où se construit l'assentiment aux thèmes structurant le débat public.

\section{NOTES}

1. Le présent article reprend, sous une forme réduite, un rapport de recherche remis à la Fondation Ford.

2. Kertzer D., Rituals, Politics and Power, Yale University Press, New Haven, 1968, p. 57-76.

3. Carré 0, L'Islam laïc, Colin, Paris, 1993.

4. Burgat F., L'islamisme au Maghreb, Paris, Khartala, 1988.

5. Al-'Ashmawi M. S. : auteur, notamment, de L'islamisme contre l'islam (traduit de l'arabe par R. Jacquemond), La Découverte/AI-Fikr, 1990.

6. « À l'université, il y a plusieurs gamâ'ât. (...) J'ai commencé à fréquenter la mosquée de l'université et j'ai vu qu'elles se disputaient la direction de la prière. Les étudiants des deux gamâ'ât ont même commencé à se donner des coups. » (Ahmad, 22 ans, section « études islamiques ", $2^{e}$ année).

7. Les propos des étudiants peuvent, de fait, être très critiques vis-à-vis de là " démocratie ", ou du moins vis-à-vis de ce que ce terme sous-entend pour eux : « Nous sommes des musulmans. La démocratie, ce n'est pas pour nous ; elle est pour les Occidentaux parce qu'elle permet la dissolution des mœurs : la mixité, les relations sexuelles en dehors du mariage, la consommation d'alcool... » (Iman, 22 ans, $2^{\mathrm{e}}$ année de sciences politiques).

8. II s'agit d'une initiative « qui se présente comme l'expression d'une "troisième force" et qui propose au ministère de l'Intérieur de tenter une médiation entre le gouvernement et les jeunes militants en révolte... » (Roussillon A., "Changer la société par le Jihâd », Le phénomène de la violence politique, Dossiers du Cedej, B. Dupret éd., Le Caire, 1994, p. 316).

9. Geertz C., « La religion comme système culturel », Essai d'anthropologie religieuse, Gallimard, 1972. 
INDEX

Mots-clés : islam, université, anthropologie, réislamisation

\section{AUTEUR}

SAADIA RADI

Cedej 